\title{
Rising up the Performance of Wireless Local Area Network by Varying Fragmentation Threshold using OPNET
}

\author{
H.S. Mewara \\ Department Of E.I.C \\ Govt. Engineering College,Ajmer India
}

\author{
Dhanwanti Manghnani \\ M.Tech. Scholar \\ Govt. Engineering College,Ajmer India
}

\begin{abstract}
As all can see that the demand of WLAN is increasing gradually, so here it is necessary to improve the performance of WLAN. There are many parameters which can directly affect the performance of WLAN like data rate, modulation scheme, buffer size, RTS threshold etc. One of them is fragmentation threshold. In this paper we are rising up the performance of WLAN by moderating the value of fragmentation threshold using OPNET as a simulation tool. By using fragmentation threshold parameter the large packets are be fragmented into smaller packets. Furthermore it can be demonstrated that by increasing the value of fragmentation threshold the throughput and load of the WLAN is increased, it also decreased the delay and media access delay of WLAN. This increased throughput and load simply implies that the performance of WLAN is enhanced. By increasing fragmentation threshold value we can also decreases the probability of discarding packets.
\end{abstract}

\section{Keywords}

WLAN, OPNET, Load, Delay, Media Access Delay, Throughput

\section{INTRODUCTION}

In the modern era the demand of wireless local area network is increasing rapidly; this wireless local area network (WLAN) is frequently replacing the wired LAN and providing the few meters connectivity within a campus[1-2]. Mobility, data access, flexibility, portability, synchronization for end users etc. these are the attracting features of WLAN. WLAN connects workstations of a local network to exchange data, information and other application without the use of cables [3]. So you can say that WLAN links multiple workstations of a campus and this WLAN permit data and application to be shared among all these workstations. Basically a WLAN [4] consist two key components (a) Access Point (b) A wireless card or network interfacing card. This access point connects all these wireless workstations to the wired network. In a WLAN it's very easy to add or remove any fix or mobile workstation and to install access point. This provides connectivity within a particular area, which is difficult in wired network. A wired LAN is a backbone to a wireless LAN i.e. wireless LAN is a supplement of a wired LAN not a complete solution .The design of WLAN IEEE802.11 [5-6] can be implemented using a powerful tool, which is called OptimumNetwork (OPNET). OPNET is an object oriented simulation tool, which provides a visualized simulation environment for network modeling. OPNET [7] is a good modeling tool which provides comprehensive technical support and maintenance assistance. OPNET provides versatility, robustness, traceability and user friendly environment.

\section{ATTRIBUTE AND MEASUREMENT UNITS}

\subsection{Fragmentation Threshold}

Here Fragmentation is specifies as a Fragmentation Threshold. When we are sending any data packet and if its size is greater than fragmentation threshold it divides the packet into small fragments. Only those packets are processed whose size is lesser than fragmentation threshold [8]. When the packet size is larger than fragmentation threshold it is divided into fragments and then forwarded and if we select the option "NONE" which simply implies fragmentation is not used. In that case if the packet size is higher than MSDU [9] than the packets will be discarded.

\subsection{Network Delay}

Network Delay represents end to end delay of all the packets that are received by the WLAN MACs [10] of all WLAN nodes and it forwards all packets to the higher layer. When the Access Point enabled this delay it includes medium access delay at the source MAC, reception of all the fragments individually, and transfers of the frames via Access point [11].

\subsection{Load}

Load indicates total bits submitted to wireless LAN layers.

\subsection{Network Throughput}

Throughput is an average rate of successful message delivery over a physical or logical link or passage through a certain network node. It is typically measured in bits per second [12].

\subsection{Media Access Delay}

Media access delay represents the global statistic for the total of queuing and contention delays of the data [12].

\section{SCENARIO AND SETTINGS}

Here we employed a simple scenario, which consist of a subnet, in this subnet there are three workstations, one access point and one server. The name of application supported profile for these workstations is subnet. These workstations support many applications like data base (light, heavy), email (light, heavy), web browsing (light, heavy) and file transfer (Heavy). Here we have made two scenarios with different fragmentation threshold values to optimize the network performance. 


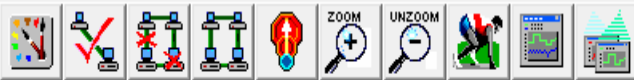

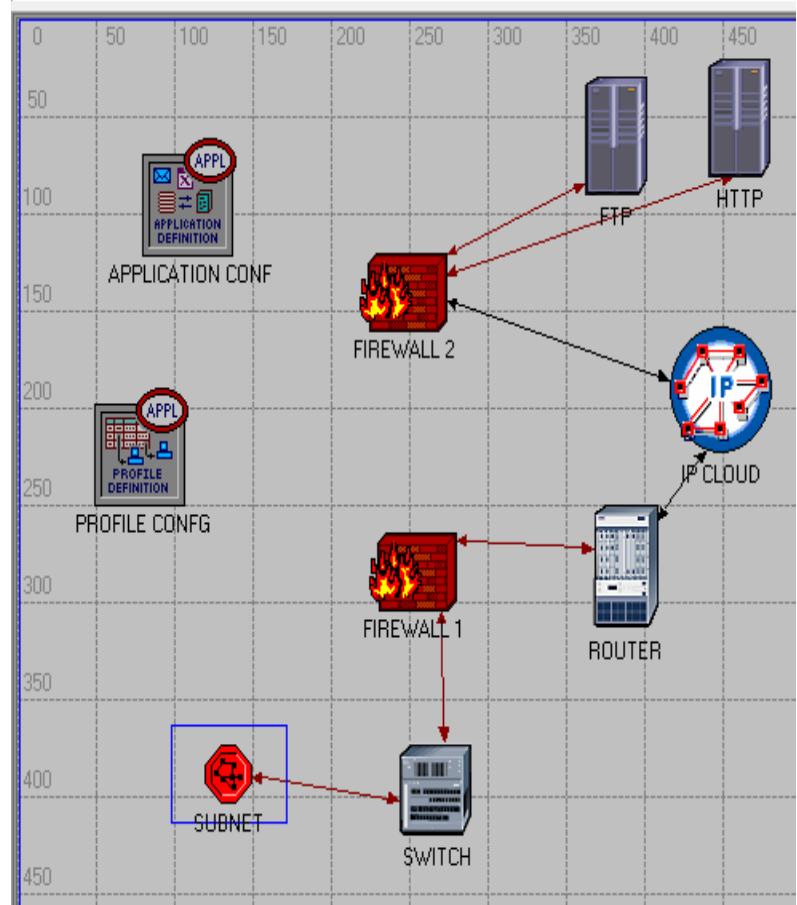

Fig 1: OPNET scenario of whole network

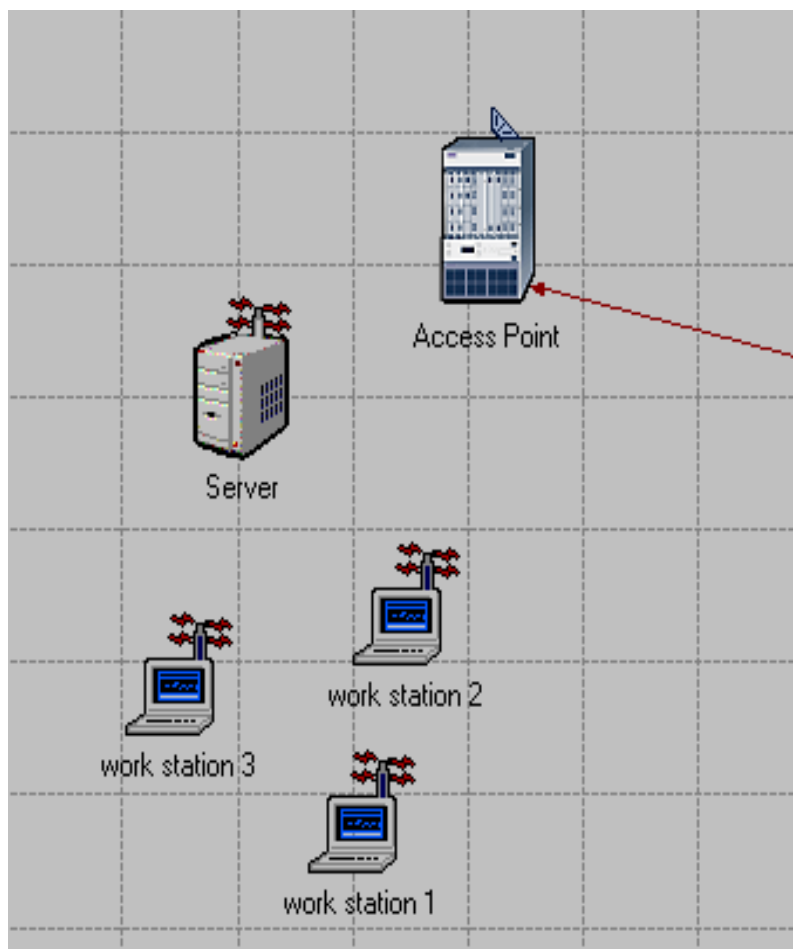

Fig 2: OPNET Scenario of subnet
Table 1. Simulation Scenario parameters

\begin{tabular}{|c|c|c|}
\hline Parameters & Scenario 1 & Scenario 2 \\
\hline $\begin{array}{c}\text { RTS } \\
\text { Threshold(Bytes) }\end{array}$ & None & None \\
\hline $\begin{array}{c}\text { Fragmentation } \\
\text { Threshold(Bytes) }\end{array}$ & 16 & 1024 \\
\hline Data rate(bps) & $11 \mathrm{Mbps}$ & $11 \mathrm{Mbps}$ \\
\hline $\begin{array}{c}\text { Physical } \\
\text { characteristics }\end{array}$ & $\begin{array}{c}\text { Frequency } \\
\text { Hopping }\end{array}$ & $\begin{array}{c}\text { Frequency } \\
\text { Hopping }\end{array}$ \\
\hline $\begin{array}{c}\text { Packet Reception } \\
\text { Power Threshold }\end{array}$ & $7.33 \mathrm{E}-14$ & $7.33 \mathrm{E}-14$ \\
\hline Short Retry limit & 7 & 7 \\
\hline $\begin{array}{l}\text { Long Retry limit } \\
\text { Access Point } \\
\text { functionality }\end{array}$ & Disabled & Disabled \\
\hline
\end{tabular}

Table 2.Wireless LAN Traffic Generation Parameters

\begin{tabular}{|c|c|}
\hline Attribute & Value \\
\hline Operation Mode & Simultaneous \\
\hline Start Time & Uniform (100,110) \\
\hline Duration (seconds) & End of Simulation \\
\hline Repeatability \\
(a)Inter Repetition \\
Time(Sec) \\
(b)No. of Repetition \\
(c)Repetition Pattern
\end{tabular}

\section{SIMULATION ANALYSIS}

The simulation analysis had been done by using OPNET IT Guru Academic edition 9.1[13]. Figure 3 shows the WLAN delay (sec) for all two scenarios, here we can see that for scenario 1 delay is around $0.0175 \mathrm{sec}$ and for scenario 2 it is $0.0045 \mathrm{sec}$. In figure 4 WLAN load (bits/sec) had been plotted for all two scenarios. From figure we can see that load for scenario 1 is around $49 \mathrm{Kbps}$ and scenario 2 it is $61 \mathrm{Kbps}$. 


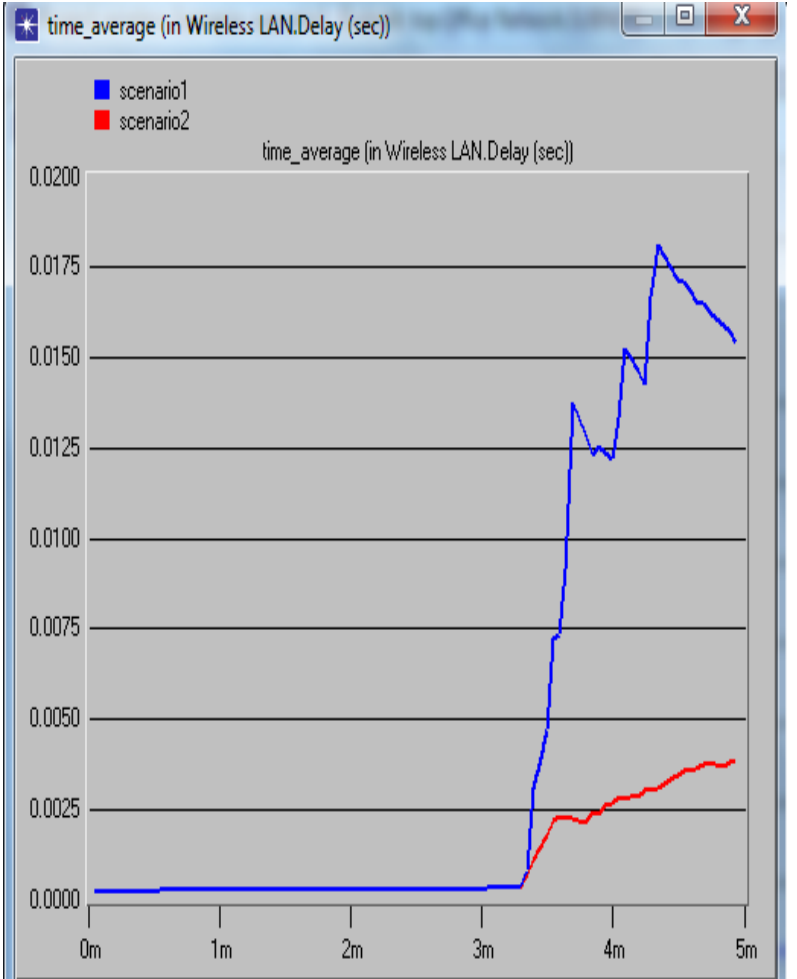

Fig 3: Wireless LAN delay (sec)

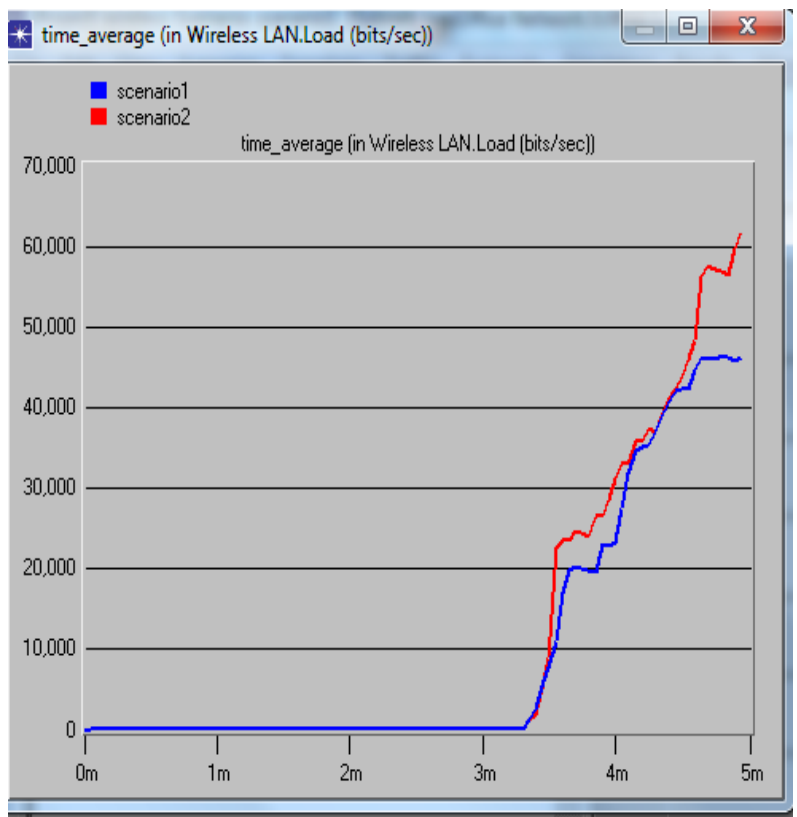

Fig 4: Wireless LAN load (bits/sec)

In figure 5 Wireless LAN media access delay had been plotted for both scenarios. For scenario 1 the media access delay is around $0.006 \mathrm{sec}$ and for scenario 2 it is $0.002 \mathrm{sec}$. Figure 6 shows the wireless LAN throughput (bits/sec). Throughput is around $25 \mathrm{Kbps}$ for scenario 1 and it is around $31 \mathrm{Kbps}$ for scenario 2 . time_average (in Wireless LAN.Media Access Delay (sec))

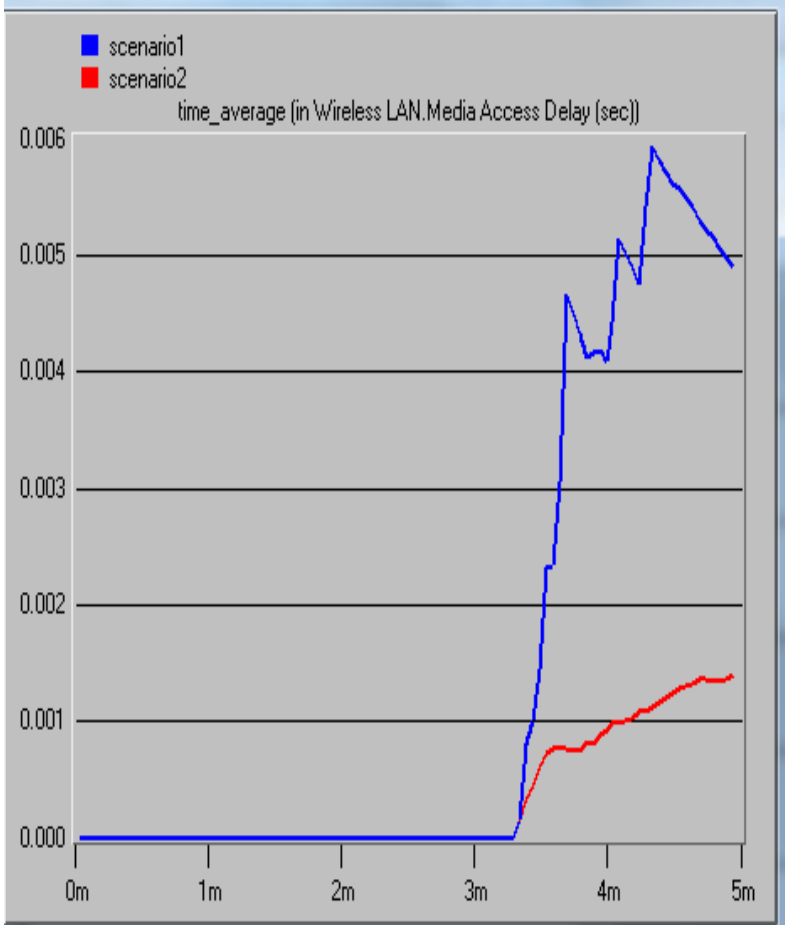

Fig 5: Wireless LAN media access delay (sec)

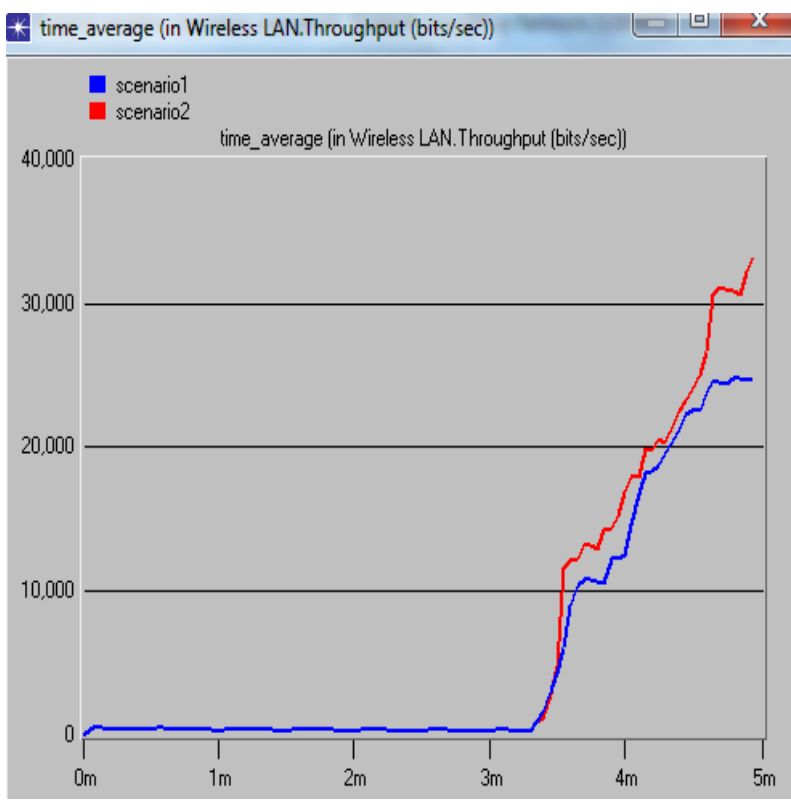

Fig 6: Wireless LAN throughput (bits/sec)

\section{CONCLUSION}

In this paper we investigated the delay, load, media access delay and throughput for two different values of fragmentation threshold. Here we can see that when the value of fragmentation threshold is increased it directly affects delay, media access delay, load and throughput. Scenario 1 having fragmentation threshold is 16 bytes offers maximum delay and media access delay and due to this the load and 
throughput is decreased. While for scenario 2 fragmentation threshold is 1024bytes offers minimum delay and media access delay and shows maximum load and throughput. Here we can conclude that fragmentation threshold is directly affects the performance of WLAN. By increasing the value of fragmentation threshold we can enhance the performance of WLAN.

\section{REFERENCES}

[1] Rappaport T., 2002.Wireless communications: Past events and a future perspective. IEEE Commun. Mag., 40:148161.

[2]Garcia, A., 2004.Networking infrastructure. eWeek, 14:5253.

[3]Brown, B., 2001.Wireless Networking's Next Big Thing. PC Mag., 21:32-34.

[4] Dr. MayyadaHammoshi, Mrs. RazanAlani, "A Proposed Model to Implement Load and Throughput of WLAN Implemented as Wi-Fi System", JCIT: Journal of Convergence Information Technology, Vol. 5, No. 7, pp. $100 \sim 111,2010$

[5] Xu, S., S.papavassiliou and S. Narayan, 2004. Layer-2 multi-hop IEEE 802.11 architecture:design and performance analysis. IEEE Proceedings of Communications, 151:460-466.

[6] Henderson, R., 2003.Host mobility for IP networks: A comparison. IEEE Network, 6:8-26.
[7] OPNET Technologies, www.opnet.com.

[8] Edarado b., E. Cooper and R. Sansom, 1993. Designing a Practical ATM LAN. IEEE Network.

[9] Borah D. K., Daga A., Lovelace G. R., and Deleon P., "Performance Evaluation of the IEEE 802.11a and b WLAN Physical Layer on the Maritain Surface", IEEE Aerospace Conference, Canada, 2005.

[10] Soliman A. Al-Wabie (2002), —The New Wireless Local Area Networks (WLAN's) Standardll, University of Maryland

[11] RajanVohra, R.S Sawhney, G.S Saini,"OPNET based wireless LAN Performance Improvisation" International Journal of Computer Applications (0975-8887), Volume 48-No.1 June 2012.

[12] SantoshKulkarni, Pratap S. Prasad, and PrathimaAgrawal. 2008. Performance enhancement of mobile ad hoc networks using nodal cooperation. In Proceedings of the 4th Annual International Conference on Wireless Internet (WICON '08). ICST (Institute for Computer Sciences, Social-Informatics and Telecommunications Engineering), ICST, Brussels, Belgium, Belgium, Article 54, 8 pages

[13] IT Guru Academic Edition (2007), OPNET Technologies www.opnet.com/university_program/itguru_academic_e dition 